\title{
AN ASSESSMENT OF THE EFFICACY OF DFMO IN BABOONS (PAPIO ANUBIS) INFECTED WITH TRYPANOSMA BRUCEI GAMBIENSE
}

\author{
A. W. MBAYA, C. O. NWOSU, M. M. ALIYU AND V. O. TAIWO
}

(Received 8, July 2008; Revision Accepted 29, September 2008)

\begin{abstract}
Infection of four baboons with Trypanosma brucei gambiense resulted in a prepatent period of $3-4$ days. Following the first appearance of $T$. $b$. gambiense parasitaemia, the animals developed trypanosomosis characterised by elevated parasite counts in the blood, fever, increased heart and respiratory rates and increased capillary refill time. The disease was also associated with pallor of visible mucous membranes, oedema, increased reticulocyte counts, progressive decline in erythrocyte indices (RBC, $\mathrm{Hb}$ and PCV), consistent monocytosis, and leucopenia due to lymphopenia and neutropenia. These clinical signs and widespread pathological changes seen in the liver, kidney, lymph nodes, heart and brain were progressive with the disease. The disease also disrupted the circadian rhythmicity of sleep and wakefulness between weeks 8 and 10 when the animals were in the classical diurnal sleepiness with 8 10 sleep episodes and nocturnal restlessness. All the infected baboons died from the attendant disease between the 8 and 10 weeks of infection. Treatment with Berenil or DFMO at 4 weeks post infection reversed most of the clinical, haematological and pathological changes, the CSF-WBC counts and cleared the parasites from the circulation of the infected baboons. However, there was relapse parasitaemia by 18 and 20 weeks respectively post infection in the groups treated with DFMO and Berenil. The results of this study suggest that baboons manifest similar clinical and pathological lesions as man infected with $T$. $b$. gambiense and might therefore be a useful model for the study of the human disease. Furthermore, the results suggest the therapeutic usefulness of DFMO in the treatment of human and animal trypanosomosis due to $T$. b. gambiense.
\end{abstract}

KEYWORDS: Sleeping sickness, Baboons, DFMO.

\section{INTRODUCTION}

Human African trypanosomosis or sleeping sickness due to Trypanosoma brucei gambiense is a reemerging complex and debilitating disease of man. It is prevalent in several areas of Central and West Africa (Solano et al., 2003; Grootenhuis, 2004). The disease is zoonotic with many wild and domestic animals being identified as potential animal reservoir hosts (Abenga and Lawal, 2005; Simo et al., 2006; Njoukou et al., 2006).

The Gambian sleeping sickness evolves through two clinically different stages; the early or haematolymphatic stage and the late or meningoencephalic stage characterised by central nervous system (CNS) disturbances (Poltera, 1985; Anonymous, 1998). Both stages terminate in death when untreated (Camara et al., 2005; Chretien et al., 2005). Presently, there is no consensus on the diagnostic criteria for the various stages and especially for specific indications for therapy and follow up when the central nervous system (CNS) is invaded (Courtioux et al., 2005; Kennedy, 2006a, b). The problem of diagnosis is further complicated by the absence of a clear understanding of the clinical and pathological features of the disease in animal models.
The major drugs for the treatment of affected individuals are suramin and melarsoprol especially when the CNS is invaded (Erhardt et al., 2006). However, as a result of the adverse and sometimes fatal reactions frequently associated with such treatments, several workers have tried DL- $\alpha$-difluoromethyl ornithine (DFMO) as a therapeutic alternative (Onyeyili and Anika, 1990; Boa et al., 1995; Khonde et al., 1995).

In this study, baboons (Papio anubis) were experimentally infected with Trypanosoma brucei gambiense ( $T$. b. gambiense) and treated with DFMO to evaluate their possible usefulness as animal models for studying the Gambian sleeping sickness.

\section{MATERIALS AND METHODS}

\section{Experimental Animals}

Sixteen adult baboons of both sexes were used for this study. They were acquired from the Sanda Kyarimi Park, Maiduguri, Nigeria. The animals were routinely de-wormed orally with pyrantel permeate (Combantrim, Pfizer Ltd., USA) and treated with oxytetracycline hydrochloride against rickettsial organisms and Diminazene aceturate (Berenil) against piroplasmosis. They were placed in squeeze cages in a

A. W. Mbaya, Department of Veterinary Microbiology and Parasitology, University of Maiduguri, P. M. B. 1069 Maiduguri, Borno State, Nigeria.

C. O. Nwosu, Department of Veterinary Microbiology and Parasitology, University of Maiduguri, P. M. B. 1069 Maiduguri, Borno State, Nigeria.

M. M. Aliyu, Department of Veterinary Medicine, University of Maiduguri, P. M. B. 1069 Maiduguri, Nigeria.

V. O. Taiwo, Department of Veterinary Pathology, University of Maiduguri, P. M. B. 1069 Maiduguri, Nigeria. 
fly-proof room. The animals were fed fresh fruits and vegetables while water was provided ad libitum. They were allowed to acclimatize to their new environment 4 weeks before the commencement of the experimental study. The Research Committee of the Faculty of Veterinary Medicine, University of Maiduguri and the Borno State Department of Wildlife approved this experiment and the animals were handled in accordance with internationally accepted principles for animal care and use.

\section{Trypanosomes}

Trypanosoma brucei gambiense (strain NITR/Abraka) was used for the study. The parasite was isolated from a human case of sleeping sickness at Abraka (southern Nigeria) in September 1990. The isolates were confirmed to be $T$. b. gambiense using the serum incubation and infectivity test (Owen and Gillete, 1992). They were maintained by serial passages in rats and produced acute and sub-acute infections with a prepatent period of $2-4$ days in donor baboons.

\section{Test drugs}

DL- $\alpha$ - difluoromethyl ornithine (DFMO) was obtained from Meril Dow Research Institute, Ohio, USA in a white crystalline form. In each case, a fresh preparation of the drug was administered for seven days at $300 \mathrm{mg} / \mathrm{kg}$ body weight as a $2 \%$ solution in drinking water. To ensure adequate consumption of the drug, it was served when the ambient temperature was relatively high and completely consumed before normal drinking water was served to the animals. Berenil (Diminazemne aceturate; Faewerke, A. G. Germany) was administered as a single intramuscular injection at $3.5 \mathrm{mg} / \mathrm{kg}$ body weight. In all cases, treatment of the experimental groups commenced at 4 weeks post infection.

\section{Experimental protocol}

The baboons were divided into four groups (A, $B, C$ and $D$ ) of four animals each. The baboons in groups $A, B$ and $C$ were each infected intravenously with $5 \times 10^{3} T$. b. gambiense in baboon blood diluted in phosphate-buffered glucose saline. The baboons in group A were treated with DFMO and group B with Berenil while those in group $C$ remained untreated. Animals in groups $D$ were uninfected and untreated and served as healthy control.

Each experimental group was housed separately and monitored daily for the development of clinical signs of trypanosomosis. Live body weight, the degree of parasitaemia and changes in haematological parameters were assessed every two weeks. The study lasted twenty weeks.

\section{Assessment of Drug Efficacy}

\section{Clinical evaluation}

Clinical parameters including rectal temperature, pulse and respiratory rates were monitored daily throughout the duration of the study.

\section{Determination of parasitaemia}

Parasitaemia was first detected by daily examination of peripheral blood using the wet mount and buffy coat techniques (Murray et al., 1983). The degree of parasitaemia was estimated every two weeks using the rapid matching technique (Herbert and Lumsden, 1976).

\section{Haematological evaluation}

Blood samples were collected every two weeks through the cephalic vein. Packed cell volume (PCV) and the numbers of red blood cells (RBC), reticulocyte, total white blood cells (WBC) and differential WBC were estimated as described by Jain (1993).

Samples of the cerebrospinal fluid (SCF) were taken by lumber puncture between the $4^{\text {th }}$ and $5^{\text {th }}$ lumber vertebrae in all the experimental baboons. The WBC and trypanosome counts were estimated every two weeks (Khonde et al., 1995).

\section{Gross and histopathological examinations}

Necropsy of dead baboons and those humanely sacrificed at the end of the study was performed and tissue samples (liver, heart, lymph nodes, spleen, kidney and lungs) collected were fixed in $10 \%$ formal saline. The tissues were embedded in paraffin wax, cut at $5 \mu$ thickness and routinely stained with haematoxylin and eosin stain for histological examination and photomicrography (Drury and Wallington, 1976).

\section{RESULTS}

\section{Parasitaemia}

Trypanosoma brucei gambiense parasitaemia was first detected in all the infected groups of baboons 3 - 4 days post infection. In the infected/untreated group, parasite counts rose sharply to a peak 10 weeks post infection (Fig. 1). Treatment with Bereni or DFMO resulted in significant reduction of parasite counts and by 8 and 10 weeks post infection, the parasites were respectively no longer detectable in blood in the two groups. Relapse parasitaemia was however noted from 18 and 20 weeks respectively post infection in the groups treated with DFMO and Berenil. 


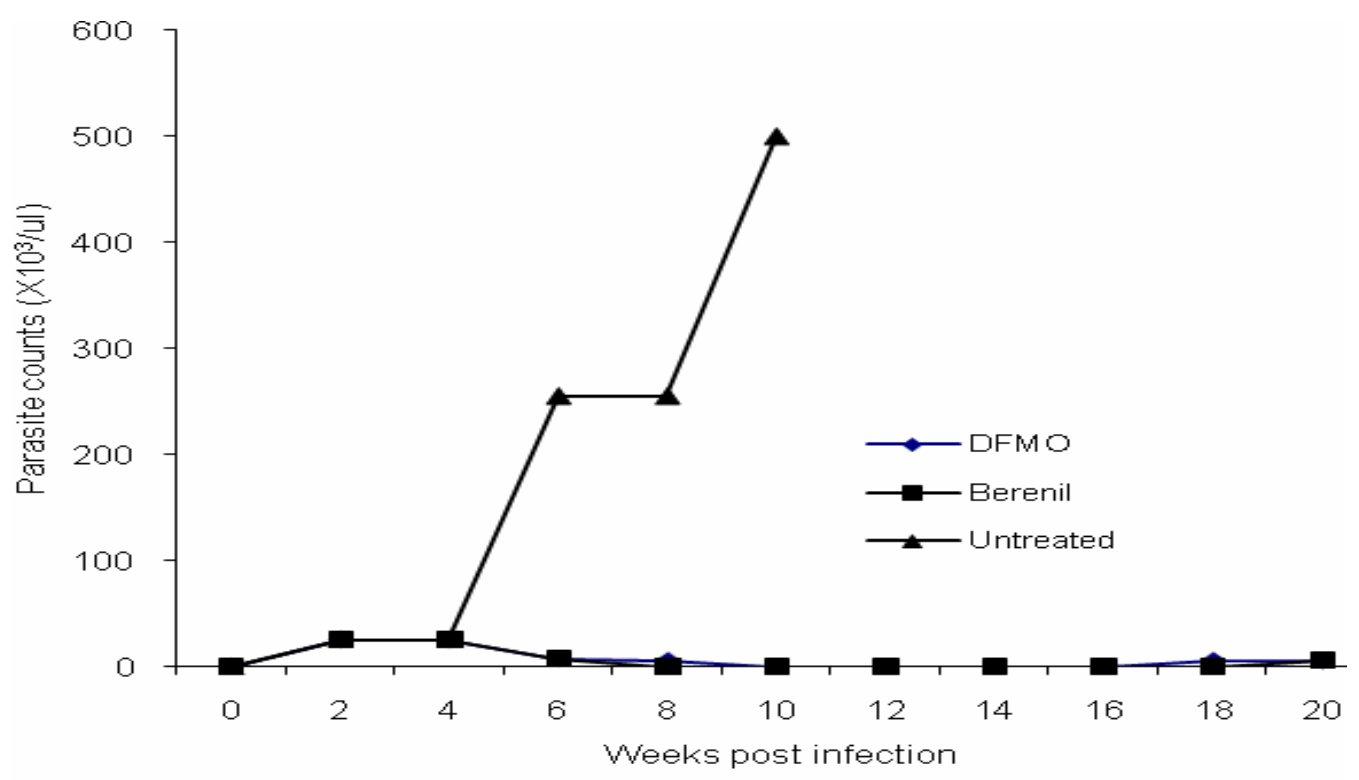

Fig. 1: Mean parasitaemia of baboons infected with T.b.gambiense and treated with DFMO or Berenil and their control

\section{Clinical observations}

Following the first appearance of parasitaemia, all the infected baboons developed clinical trypanosomosis characterised by fever and increased heart and respiratory rates with prolonged capillary refill time. In the infected/untreated group, these clinical signs were progressive and from 8 weeks post infection there were signs of fatigue, peri-orbital oedema and oedema of the hind quarters, bilateral submandibular lymphadenopathy and pallor of mucous membranes. Two baboons in this group convulsed and during this period there was somnolence (hypersomnia) with $8-10$ sleep episodes in day time and nocturnal insomnia. All the baboons in this group died from the infection between week 8 (one baboon) and week 10 (three baboons).

Besides the initial signs of fever, treatment with either DFMO (Group A) or Berenil (Group B) suppressed the manifestation of the clinical signs of the disease and none of the baboons in either of this group died from the infection during the study period.

\section{Haematological observations}

Following the first appearance of parasitaemia, there was precipitous decline in the RBC, PCV and $\mathrm{Hb}$ values of all the infected baboons (Figs. 2 and 3 ). The decline in the values of these parameters continued unabated until death of all the baboons in the infected/untreated group by 10 weeks post infection. On the other hand, treatment with DFMO or Berenil by week 4 post infection resulted in a decline in the values of the erythrocyte indices or their increase towards preinfection values for the rest of the study period.
Following infection, the reticulocyte values of all the infected baboons showed a steady increase to a peak by 10 weeks post infection corresponding to the lowest level of the other erythrocyte indices and the death of all the animals in the infected/untreated group (Fig. 3). In those groups treated with Berenil or DFMO, reticulocyte numbers continually declined from week 12 but failed to reach their pre-infection levels at the end of the study. In the healthy control group, reticulocyte counts did not vary significantly from their pre-infection levels throughout the study period.

Leucocyte counts of the healthy control group remained within their pre-infection values throughout the study (Fig. 4). On the other hand, there was a gradual decline in the leucocyte numbers in all the infected groups from week 8 post infection. In the group treated with DFMO and Berenil, leucocyte numbers reached their lowest levels by 10 and 12 weeks respectively post infection following which there was a continuous increase towards pre-infection levels. These attempts toward recovery of the values were further aborted following the development of relapse parasitaemia in both groups. The leucopeania was mainly due to lymphopenia and neutropenia. There was however, a consistent monocytosis in all the infected baboons, including those treated with DFMO or Berenil.

Cerebrospinal fluid leucocyte (CSF - WBC) counts remained within the pre-infection values in the healthy control group throughout the study (Fig. 4). On the other hand, there was a sharp increase in the CSF WBC counts of all the infected baboon groups to a peak at week 10 when all the infected/untreated animals died from the infection. Treatment with Berenil or DFMO resulted in a sharp and continuous decline in the CSF WBC counts towards pre-infection values for the rest of the study period. 

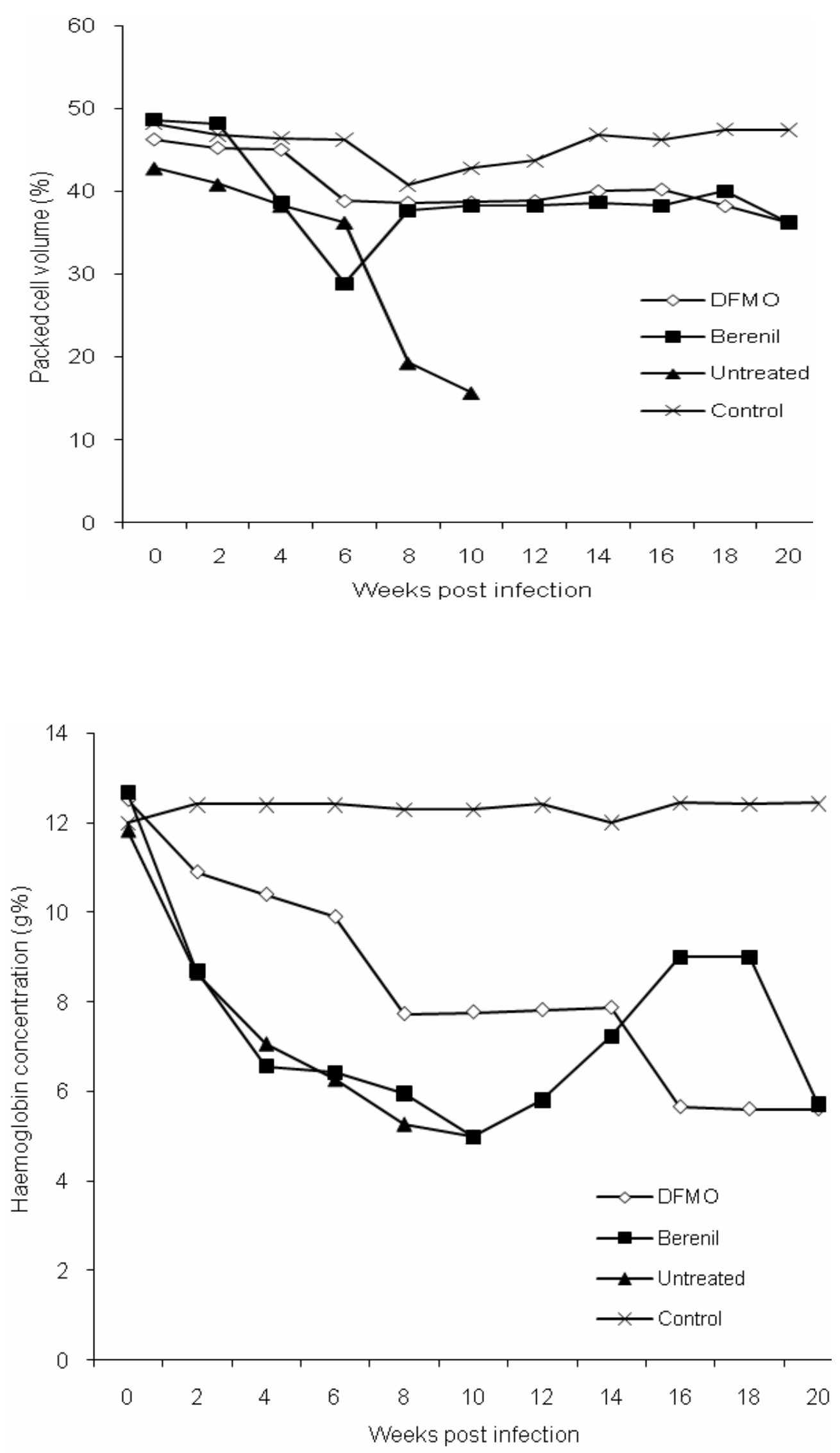

Fig. 2. Mean PCV and $\mathrm{Hb}$ concentration of baboons infected with $T$. b. gambiense and treated with DFMO or Berenil and their control 

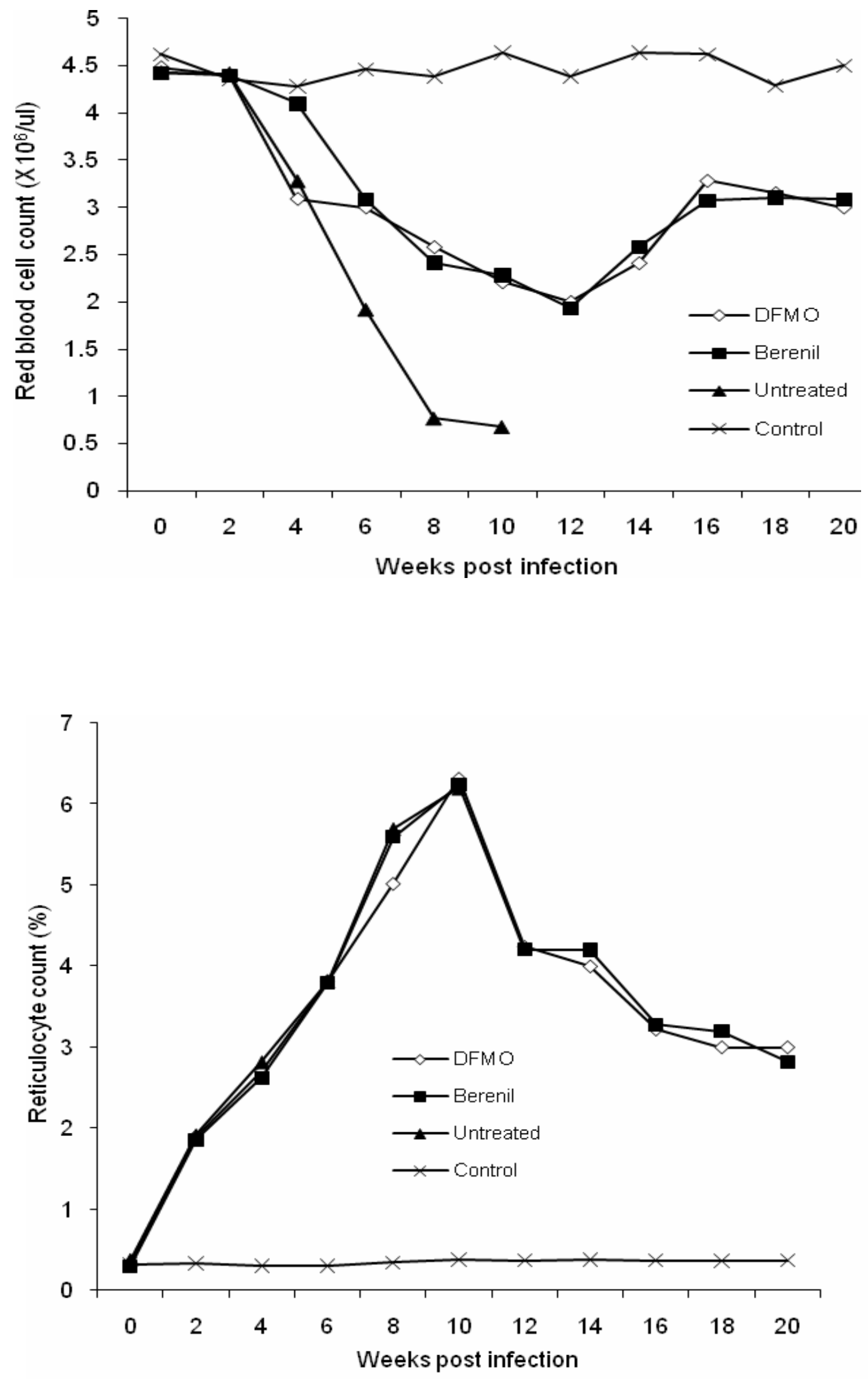

Fig. 3. Mean RBC and reticulocyte counts of baboons infected with T. b. gambiense and treated with DFMO or Berenil and their control 

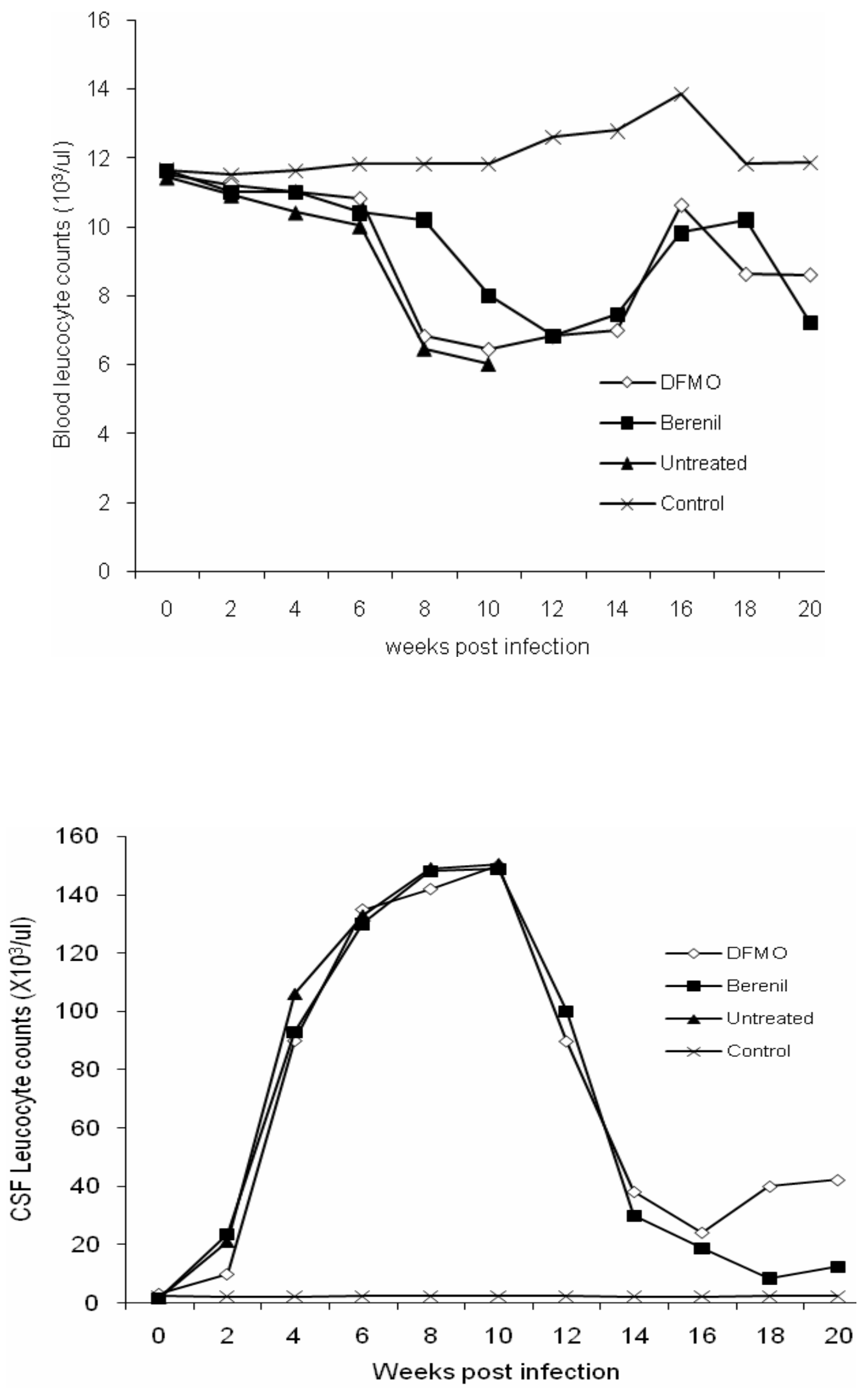

Fig. 4. Mean blood and CSF leucocyte counts of baboons infected with $T . b$. gambiense and treated with DFMO or Berenil and their control 


\section{Necropsy findings}

The gross lesions observed at necropsy in the baboons that died from the infection or those humanely sacrificed at the end of the study include varying severities of hepatomegaly, splenomegaly, submandibular lymphadenopathy, serous atrophy of pericardial, abdominal and perirenal fat and congestion of meningeal blood vessels.

Histological lesions included depletion and necrosis of lymphocytes and lymphophagocytosis by numerous macrophages and haemosiderosis in lymph nodes and spleen (Plate 1); pulmonary congestion and oedema, thickened inter-alveolar septa and mononuclear cellular infiltration and haemosiderosis in the lungs; sinusoidal congestion, periportal and centrilobular vacuolar degeneration and necrosis of hepatocytes, and mononuclear cell aggregations (Plate 2); multifocal areas of hyaline degeneration of myocardial fibres; widespread glomerular and tubular degeration and the presence of proteinaceous casts in renal cortical tubulaes (Plate 3) and meningeal congestion and non-suppurative meningo-encephalitis. These lesions were generally more severe in the infected/untreated baboons but only mild to moderate in those treated with either Berenil or DFMO.

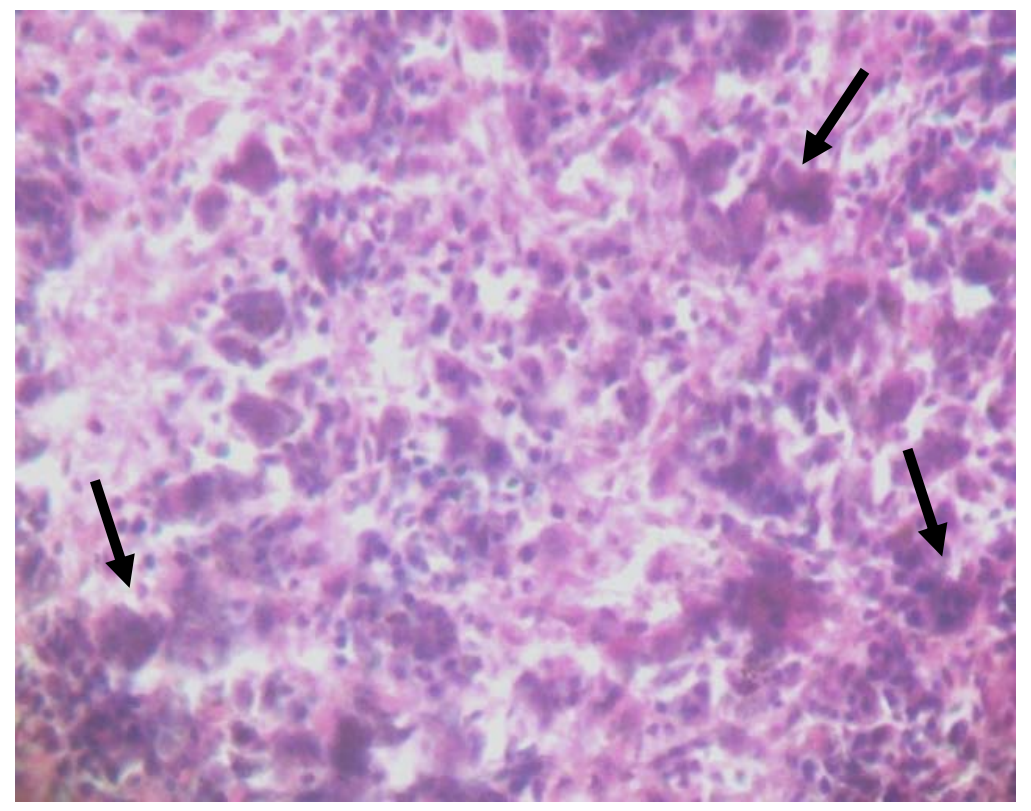

Plate 1: Spleen of baboon (Papio anubis) infected with Trypanosoma brucei gambiense and untreated showing widespread lymphoid necrosis and lymphophargocytosis by numerous macrophages (arrows) (H\&E; x450)

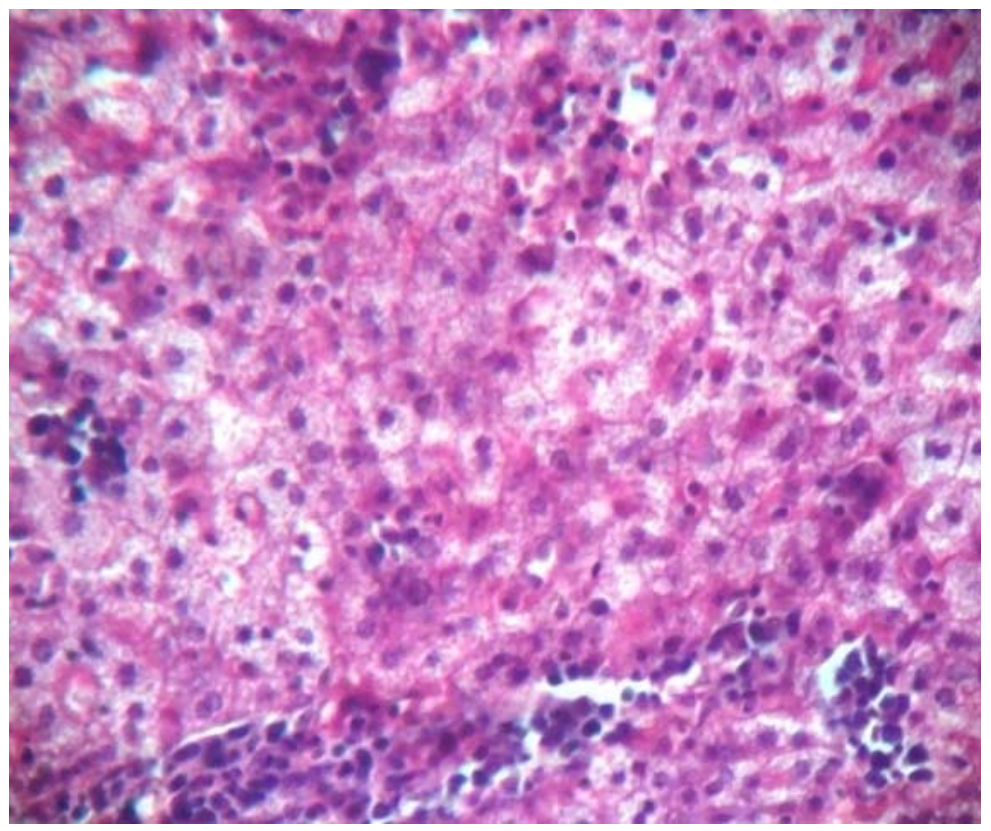

Plate 2: Liver of baboon (Papio anubis) infected with Trypanosoma brucei gambiense and treated with Berenil囚 showing widespread vacuolar degeneration and necrosis of hepatocytes, and mononuclear cell aggregations (H\&E; x500) 


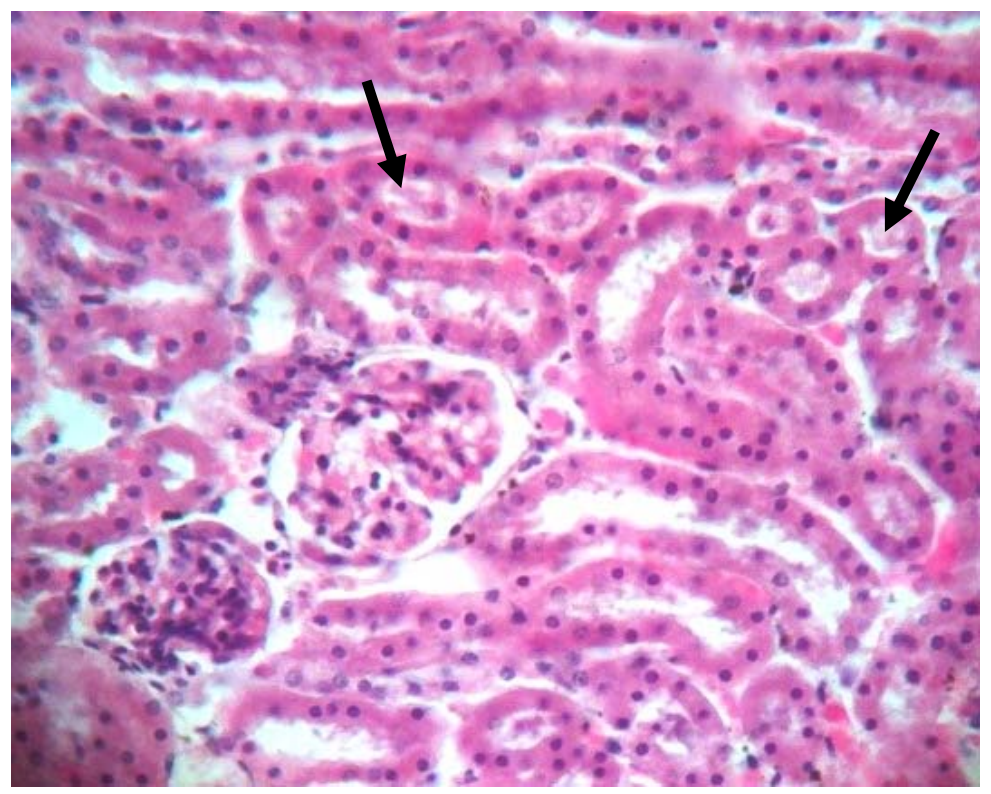

Plate 3: Kidney of baboon (Papio anubis) infected with Trypanosoma brucei gambiense and treated with DMFO showing glomerular and tubular degeneration and presence of proteinaceous casts in cortical tubules (arrows) (H\&E; x450)

\section{DISCUSSION}

The results of this study showed that DFMO at the dose of $300 \mathrm{mg} / \mathrm{kg}$ body weight used in this study reversed the morbidity and mortality of baboons infected with $T . b$. gambiense. The observed reversal of the clinical manifestations, haematological and pathological changes with a clearance of the parasites from the circulation of the infected baboons is consistent with earlier reports in humans naturally infected with the parasite (Spinazzola et al., 1990; Boa et al., 1995; Khonde et al., 1995).

The acute course of the infection in the baboons mimicked the course of experimental $T . \quad b$. brucei infection in animals (Losos and Ikede, 1972; Nwosu and Ikeme, 1992). Similar observations were made in natural infections of man (Ford, 1971) and experimental infections of vervet monkeys (Abenga and Anosa, 2006) with the parasite. The same strain of parasite had earlier produced similar acute manifestations in human outbreaks of sleeping sickness at Abraka in southern Nigeria (Edeghere et al., 1989; Enwezor and Ukah, 2000). However, the acute nature of the present study differed significantly from the typical chronic disease usually seen in man (Picozzi et al., 2005).

Anaemia is the commonest feature of African animal and human trypanosomosis (Anosa, 1983; Losos and Ikede, 1972; Nwosu and Ikeme, 1992). In the present study, anaemia associated with significant reductions in the values of erythrocyte indices (RBC, $\mathrm{PCV}$ and $\mathrm{Hb}$ ) was recorded in the infected baboons. Abenga and Anosa (2004) made the same observations in vervet monkeys experimentally infected with $T . b$. gambiense. According to Anosa (1988), the anaemia of trypanosomosis is related to the severity of the attendant disease; acute diseases being associated with precipitous drops in erythrocyte indices without abatement until death of the infected animals. A similar pattern of anaemia occurred during this study, as the reductions in erythrocyte indices were more precipitous and continued until death of the untreated baboons. However, treatment with Berenil or DFMO and the consequent clearance of the parasites from the circulation reversed this trend with eventual appreciation in the values of these parameters towards their preinfection levels.

There was evidence of erythropoietic response in the femoral marrow as manifested by the consistent reticulocytosis recorded in the infected baboons. However, reticulocyte response was more marked in the early part of the disease when the baboons also manifested greater anaemia than after treatment with DFMO or Berenil. Earlier reports showed that erythropoietic responses in animals infected with trypanosome species was related to the degree of anaemia (Igbokwe and Mohammed, 1991; Nwosu and Ikeme, 1992; Abenga and Anosa, 2006).

The bilateral inflammation of the frontal lobes of the brain as noted in this study were associated with leuco-encephalitis and have been reported in human infections with trypanosome species (Spinazzola et al., 1990). According to Pentreath (1995), the breakdown of the choroids plexus during the later part of trypanosomosis compromises the blood-brain barrier with a resultant movement of the parasites into localised areas of the brain. A similar pathogenesis may have occurred during this study as the intra peritoneal injection of homogenates of the brain from the relapsed baboons produced parasitaemia in recipient albino rats. On the other hand, homogenates of other tissues including the liver, heart, kidney and lymph nodes of the same baboons failed to produce parasitaemia in recipient albino rats after 21 days. At this point, treatment with DFMO or Berenil had cleared the 
parasites from the circulation and the CSF and had significantly reduced the CSF - WBC counts. Similar observations were made in dogs infected with $T . b$. brucei (Chukwu et al., 1990) and in human infections of T. b. gambiense (Nantulya et al., 1992). Consequently, the parasites responsible for the relapse infection must have originated from the localised areas of the brain.

At the meningo-encephalitis (ME) stage, African sleeping sickness has been reported to cause a disruption of the circadian rhythmicity of sleep and wakefulness (Buguet et al., 1989; 2005). Similar observations were made in the present study. The baboons were in the ME stage between the $8^{\text {th }}$ and $10^{\text {th }}$ weeks of infection and manifested 8 - 10 sleep episodes during the day thus conforming with the classical diurnal sleepiness and nocturnal wakefulness earlier reported in man (Ford, 1971; Buguet et al., 1989, 2005) and vervet monkeys (Abenga and Anosa, 2006) infected with the same parasite.

\section{CONCLUSION}

In conclusion therefore, the results of this study show that baboons manifest similar clinico-pathological features as man infected with $T$. b. gambiense suggesting the possible usefulness of the baboon as a good model for the study of the Gambian sleeping sickness. The results further suggest the efficacy of DFMO in the treatment of the Gambian sleeping sickness. However, the relapse parasitaemia observed from week 18 post infection of the treated animals constitute a serious draw back in the usefulness of this drug for the routine treatment of the disease.

\section{ACKNOWLEDGEMENTS}

The authors are grateful to the staff of Sanda Kyarimi Park, Maiduguri, Nigeria for donating some of the baboons and providing the squeeze cages for the study and to Professors G. O. Egwu and P. A. Onyeyili for supplying the DFMO.

\section{REFERENCES}

Abenga, J. N. and Anosa, V. O., 2006. Clinical studies on experimental Gambian trypanosomosis in vervet monkeys. Veterinarski Arhiv, 76: 11 - 18.

Abenga, J. N. and Lawal, I. A., 2005. Implicating roles of animal reservoir hosts in the resurgence of Gambian trypanosomiasis (Sleeping Sickness). African Journal of Biotechnology, 4: 134 - 137.

Anonymous, 1998. Control and surveillance of African trypanosomosis. World health organisation Technical Report series 881.

Anosa, V. O., 1983. Mammalian blood cells in health and in trypanosomiasis. Tropical Veterinarian, 1: $177-199$.

Anosa, V. O., 1988. Haematological and biochemical changes in human and animal trypanosomiasis. Part II. Rev. Elev. Med Vet. Pays trop., 41: 151 $-164$.
Boa, Y. F., Mouanga, A., Assi, A. B., Ettien, F., Diagana, M., Oussi, B. and Giordano, G., 1995. Gambiense African trypanosomosis: a seven-day course of DFMO could be enough. OAU/STRC, 18(9123): $104-106$.

Buguet, A., Gati, R., Severe, J. P., Develeroux, M., Bogui, and Londsdorfer, J., 1989. Twenty four hour polydomnographic evaluation of sleeping sickness. Electroencephalography and Clinical Neurophysiology, 72: 471-478.

Buguet, A., Blisser, S., Josenando, T., Chapotot, F. and Cespuglio, R., 2005. Sleep structure: a new diagnostic tool for stage determination in sleeping sickness. Acta Tropica, 93: 107 - 117.

Camara, M. K., Kaba, D., KagbaDouno, M., Sanon, J. R., Ouendeno, F. F. and Solano, P., 2005. Human African trypanosomiasis in a mangrove zone in the Republic of Guinea: epidemiological and clinical characteristics in two adjacent outbreak areas. Medecine Tropicale, 65: 155-161.

Chretien, J. P. and Smoak, B. L., 2005. African trypanosomiasis: changing epidemiology and consequences. Current Infectious Diseases Report, 7: $54-60$.

Chukwu, C. C., Anene, B. M., Onuchukwusi, K. O. and Anika, S. M., 1990. Relapse infection after chemotherapy in dogs experimentally infected with Trypanosoma brucei. J. Small Anim. Practice., 31: 141-144.

Courtioux, B., Blisser, S., M'Belesso, P., Ngoungou, E., Girard, M., Nangouma, A., Josenando, T., JauberteauMarchan, M. O. and Bouteille, B., 2005. Dot enzymelinked immunosorbent assay for more reliable staging of patients with human African trypanosomiasis. Journal of Clinical Microbiology, 43: $4789-4795$.

Drury, R. A. B. and Wallington, E. A., 1976. Carleton's histological techniques. $4^{\text {th }}$ edition, Oxford University press, London. Pp. $21-70$.

Ehrhardt, S., Lippert, U., Burchard, G. D. and Sudeck, H., 2006. Orchitis as an unusual manifestation of human African trypanosomiasis. Journal of Infection, 52: e31 - e33.

Enwezor, F. N. C. and Ukah, J. C. A., 2000. Advanced trypanosomiasis (sleeping sickness) in a child: case report. Nigerian Journal of Parasitology, 2: $143-146$.

Ford, J., 1971. The role of the trypanosomiasis in African ecology: a study of the tsetse-fly problem. Clarendon Press, Oxford, pp 65-71.

Grootenhuis, J. G., 2004. The role of the African wildlife in the epidemiology of cattle trypanosomosis. Parasitology, 98: $367-380$. 
Herbert, W. J. and Lumsden, W. H. R., 1976. Trypanosoma brucei: a rapid matching technique for estimating the hosts parasitaemia. Experimental Parasitology, 40: 427 - 431.

Igbokwe, I. O. and Mohammed, A., 1991. The reticulocyte response to the anaemia in goats caused by experimental Trypanosoma brucei infection. Veterinary Research Communications, 15: $373-377$.

Jain, N. C., 1993. Essentials of veterinary haematology. Lea and Febiger, Philadelphia, 417.

Kennedy, P. G., 2006a. Human African trypanosomiasis - neurological aspects. Journal of Neurology, 253: $411-416$.

Kennedy, P. G. F., 2006b. Diagnostic and neuropathogenesis issues in human African trypanosomiasis. International Journal for Parasitology, 36: $505-512$

Khonde, N., Loko, L., Mpia, B. and Pepin, J., 1995. Open trial of a seven-day course of DFMO among 32 patients with relapsing $T . b$. gambiense trypanosomosis. OAU/STRC, 18: 103.

Losos, G. J. and Ikede, B. O., 1972. Review of the pathology of the diseases in domestic and laboratory animals caused by Trypanosoma congolense, $T$. vivax, T. brucei, $T$. rhodesiense and $T$. gambiense Veterinary Pathology Supplement, 9: 1 - 71 .

Murray, M., Trail, J. C. M., Turner, D. A. and Wissocqi, Y., 1983. Livestock productivity and trypanotolerance: network training manual. International Livestock Centre for Africa (ILCA), Addis Ababa, Ethiopia, pp. $6-28$.

Nantulya, V. M., Doua, F. and Molisho, S., 1992. Diagnosis of Trypanosoma brucei gambiense sleeping sickness using an antigen detection enzyme-linked immunosorbent assay. Transactions of the Royal Society for Tropical medicine and Hygiene, 86: $42-45$.

Njoukou, F., Laveisere, C., Simo, G., Nkinin, S., Grebaut, P., Cuny, G. and Herder, S., 2006. Wild fauna as a potential animal reservoir for Trypanosoma brucei gambiense in Cameroon. Infection, Genetics and Evolution, 6: 147 - 153.

Nwosu, C. O. and Ikeme, M. M., 1992. Parasitaemia and clinical manifestations in Trypanosma brucei infected dogs. Rev. Elev. Med. Vet. Pays Trop., 45: $273-277$.
Onyeyili, P. A. and Anika, S. M., 1990. Chemotherapy of Trypanosoma brucei brucei infections: use of DFMO, diaminazene aceturate alone and in combinations. J. Small Anim. Pract., 30: 305 310.

Owen, J. S. and Gillete, M. P. I., 1992. Cytotoxic effects of human plasma: insight and confusion from studies in cirrhotic patients, in baboons and transgenic mice. Annals de la Societe Belgrade Medicine Tropicale, 72: 94 - 95.

Pentreath, V. W., 1995. Trypanosomosis and the nervous system: pathology and immunology. Tropical Medicine and Hygiene, 89: 9-15.

Picozzi, K., Fevre, E. M., Odiit, M., Carrington, M., Eisler, M. C., Maudlin, I. and Poltera, A. A., 2005. Pathology of human African trypanosomosis with reference to experimental African trypanosomosis and infections of the central nervous system. Brit. Med. Bull., 41: 169 - 174.

Simo, G., Asonganyi, T., Nkinin, S. W., Njiokou, F. and Herder, S., 2006. High prevalence of Trypanosoma brucei gambiense group I in pigs from the Fontem sleeping sickness focus in Cameroon. Veterinary Parasitology, 139: 57 - 66.

Solano, P., Dela Roqoes, S. and Duvalet, G., 2003. Biodiversity of trypanosomes pathogenic for cattle and their epidemiological importance. Ann. Soc., 68: 169 - 171.

Spinazzola, F., D’Amato, C., Demartino, et al., 1990. A case of $T$. b. gambiense meningoencephalalitis: clinical, immunological, therapeutic and diagnostic aspects. Giornale di malathieninfettive Parasissitarie, 42: 231 - 234. 